\title{
Calcitonin gene-related peptide has protective effect on brain injury induced by heat stroke in rats
}

\author{
CHENG-XIANG LU ${ }^{1,2}$, TING QIU ${ }^{3}$, ZHI-FENG LIU ${ }^{4}$, LEI SU$^{1}$ and BIAO CHENG ${ }^{5}$ \\ ${ }^{1}$ Department of Intensive Care Unit, Affiliated General Hospital of Guangzhou Military Command of \\ Southern Medical University, Guangzhou, Guangdong 510010; Departments of ${ }^{2}$ Intensive Care Unit and \\ ${ }^{3}$ Neurology, Zhongshan Hospital Xiamen University, Xiamen, Fujian 361004; ${ }^{4}$ Department of Intensive Care Unit, \\ General Hospital of Guangzhou Military Command; ${ }^{5}$ Department of Plastic Surgery, Affiliated General Hospital of \\ Guangzhou Military Command of Southern Medical University, Guangzhou, Guangdong 510010, P.R. China
}

Received November 10, 2016; Accepted June 22, 2017

DOI: $10.3892 /$ etm.2017.5126

\begin{abstract}
Heat stroke often leads to multiple organ dysfunction syndrome (MODS) with a neurological morbidity of $30 \%$. Current studies suggested that pathophysiological responses to heat stroke may be due to a systemic inflammatory response syndrome and a series of peptidergic nerve reactions. The mechanisms underlying the high neurological morbidity in heat stroke have remained largely elusive. In recent years, calcitonin gene-related peptide (CGRP) has been considered to have a positive role in central nervous system injury. The present study investigated the influence of CGRP on brain injury induced by heat stroke. A rat model of heat stroke was established in a pre-warmed artificial climate chamber with a temperature of $35.5 \pm 0.5^{\circ} \mathrm{C}$ and a relative humidity of $60 \pm 5 \%$. The rectal core temperature (Tc) was monitored. Heat stress was halted at a Tc of no more than $41^{\circ} \mathrm{C}$ A bolus injection of CGRP was administered to each rat in the HS+CGRP group and a bolus injection of CGRP8-37 was administered to each rat in the HS+CGRP8-37 group after heat stress. After $2 \mathrm{~h}$, electroencephalograms were recorded and the pathological morphology of brain tissue as well as brain cell apoptosis and caspase- 3 protein levels in the brain were measured. The EEG of rats in the HS+CGRP group was characterized by a short- to long-term $\alpha$-wave and low-voltage $\beta$-waves as well
\end{abstract}

Correspondence to: Dr Biao Cheng, Department of Plastic Surgery, Affiliated General Hospital of Guangzhou Military Command of Southern Medical University, 111 Liu Hua Road, Guangzhou, Guangdong 510010, P.R. China

E-mail:958028065@qq.com

Dr Lei Su, Department of Intensive Care Unit, Affiliated General Hospital of Guangzhou Military Command of Southern Medical University, 111 Liu Hua Road, Yuexiu, Guangzhou, Guangdong 510010, P.R. China

E-mail: mynamesulei@sina.com

Key words: heat stroke, calcitonin gene-related peptide, CGRP8-37, electroencephalogram, apoptosis, caspase-3 as a large amount of intermittent $\delta$ - and $\theta$-waves. Compared with the HS group, the $\theta$-wave decreased and the $\alpha$-wave increased significantly $(\mathrm{P}<0.05)$. Slight pathological damage of nerve cells appeared in the HS+CGRP group. Greater damage was observed in HS+CGRP8-37 group with neural cell shrinkage, volume reduction, nuclear pyknosis, disappearance of part of the nuclear membrane and cell necrosis. In the HS+CGRP group, apoptotic cells and caspase-3 protein in the brain were significantly decreased when compared with those in the HS group $(\mathrm{P}<0.05)$, while they were significantly increased in the HS+CGRP8-37 group ( $<<0.05$ vs. HS group). The results of the present study reflected that CGRP has a protective effect on early-stage brain injury induced by heat stroke in rats.

\section{Introduction}

Heat stroke is a life-threatening acute condition characterized by a rapidly increasing core body temperature and central nervous system injury. Brain damage is a common manifestation of heat stroke and the high neurological morbidity observed in heat stroke has been considered secondary to multiple organ dysfunction syndrome (MODS) $(1,2)$. The mechanism is complex and involves a series of peptidergic nerve reactions (3). Calcitonin gene-related peptide (CGRP) is a novel endogenous neural active peptide, which was identified by a gene recombination technique. It not only has an important role in vasodilation and nerve conduction, but is also closely associated with the occurrence and development of brain injury $(4,5)$. Previous studies have demonstrated that CGRP significantly increases the cerebral blood flow and reduces injury of ischemic neurons in hypoxic-ischemic brain injury (6). After scald and cold stimulation, ultraviolet irradiation and water immersion stress, the expression of CGRP was also significantly increased (7-9). Therefore, the present study hypothesized that CGRP also has a protective role in brain injury induced by heat stroke. However, to date, only few results have been reported in this field. The present study aimed to explore the effects of CGRP on brain injury induced by heat stroke by constructing a model of heat stroke and injecting CGRP and its antagonist through the carotid artery. 


\section{Materials and methods}

Animals. Male Wistar rats ( $\mathrm{n}=20$, weight, 200-230 g; age, 10-12 weeks; Southern Medical University, Guangzhou, China) were maintained under controlled environmental conditions (12-h light/dark cycle; humidity, $35 \pm 5 \%$; temperature, $25^{\circ} \mathrm{C}$ ) at the Experimental Animal Center of Southern Medical University (Guangzhou, China) and were given free access to standard laboratory chow and water. Animal procedures were approved by the Animal Care and Use Committee of Southern Medical University (Guangzhou, China) and the experiment was performed according to the Guidelines for Animal Care of Southern Medical University (Guangzhou, China).

Preparation of the heat stroke model and intervention. A total of 20 rats, housed for $6 \mathrm{~h}$ at an ambient temperature of $25 \pm 0.5^{\circ} \mathrm{C}$ with a humidity of $35 \pm 5 \%$, were randomly divided into four groups of 5 animals each: Control group; HS group, heat stroke; HS+CGRP group, heat stroke and injection of CGRP (Abcam, Cambridge, MA, USA); HS+CGRP 8-37 group, heat stroke and injection of CGRP8-37 (Sigma-Aldrich; Merck KGaA; Darmstadt, Germany), a specific antagonist of CGRP receptor. Prior to establishment of the model, an intraperitoneal injection of sodium pentobarbital $(50 \mathrm{mg} / \mathrm{kg}$; Sigma-Aldrich; Merck KGaA) was applied to abolish the corneal reflex and pain reflex. The carotid artery of the rat was cannulated with a trocar (24-gauge) for drug injection. A unipolar lead with scalp acupuncture was used for electroencephalogram (EEG) examination. Acupuncture needles were inserted in the epicranium of rat's bilateral temples. Rats in the control group were maintained at $25 \pm 0.5^{\circ} \mathrm{C}$ and a humidity of $35 \pm 5 \%$. Rats from the other three groups were placed in a pre-warmed incubator at $35.5 \pm 0.5^{\circ} \mathrm{C}$ and a relative humidity of $60 \pm 5 \%$ in the absence of food and water. The rectal core temperature $(\mathrm{Tc})$ was continuously monitored with a rectal thermometer. Rats were removed from the incubator and allowed to cool at an ambient temperature of $25 \pm 0.5^{\circ} \mathrm{C}$ when the Tc reached $41^{\circ} \mathrm{C}$. Each rat in the HS+CGRP group received a bolus injection of CGRP $(2 \mu \mathrm{g} / \mathrm{ml}, 0.5 \mathrm{ml}$; Abcam) through carotid artery trocar after heat stress. In the HS+CGRP8-37 group, each rat received a bolus injection of CGRP8-37 (30 nmol/kg, $0.5 \mathrm{ml}$; Sigma-Aldrich; Merck KGaA) after heat stress. Rats in the HS group and control group received a bolus injection of normal saline $(0.5 \mathrm{ml})$. At $2 \mathrm{~h}$ after heat stress, all rats received scalp EEG examination (duration, $30 \mathrm{~mm} / \mathrm{s}$; gain, $0.5 \mathrm{~cm}=50 \mu \mathrm{V}$ ) and were then subjected to further histopathological analysis and index detection.

Brain tissue sampling. Rats were anesthetized by intraperitoneal injection of urethane and then sacrificed by decollation. Brain tissues were obtained during immediate autopsy in all animals. Tissues for hematoxylin and eosin (H\&E) staining and terminal deoxynucleotidyltransferase-mediated deoxyuridine triphosphate nick end labelling (TUNEL) were fixed in $10 \%$ formalin and embedded into paraffin blocks. Brain tissue sections $(3 \mathrm{~mm})$ were collected for western blot analysis.

Histopathological analysis. Paraffin-embedded tissues were sectioned at $3-\mu \mathrm{m}$ thickness and stained with $\mathrm{H} \& \mathrm{E}$ for microscopic evaluation at a magnification of $\mathrm{x} 400$. The extent of brain injury was evaluated by two certified pathologists in a blinded manner.

TUNEL assay. Paraffin-embedded tissues were sliced into frozen sections using a freezing microtome (Leica Microsystems, Wetzlar, Germany). The sections on the slides were conventionally de-waxed with xylene, rehydrated with graded ethanol and incubated in 3\% hydrogen peroxide methanol at room temperature for $20 \mathrm{~min}$, washed with $\mathrm{PBS}$ for $30 \mathrm{~min}$, permeabilized with $0.1 \%$ Triton $\mathrm{X}-100$ for $2 \mathrm{~min}$ at $4^{\circ} \mathrm{C}$, washed twice with PBS, and then incubated with TUNEL staining mixture (Nanjing KeyGen Biotech, Nanjing, China) at $37^{\circ} \mathrm{C}$ in the dark for $1 \mathrm{~h}$. Subsequently, samples were incubated with 3,3'-diaminobenzidine in the dark for $2 \mathrm{~min}$. The samples were then washed three times with PBS, counterstained with hematoxylin and mounted with glycerol. Apoptosis was observed using a microscope (Olympus, Tokyo, Japan) and three visual fields of view were randomly selected to count the cells with positive staining.

Western blot analysis. Brain sections were lysed with radioimmunoprecipitation assay buffer, which contained $50 \mathrm{mM}$ Tris- $\mathrm{HCl}, 150 \mathrm{mM} \mathrm{NaCl}, 1 \%$ Nonidet P-40, $0.25 \%$ Na-deoxycholate and $1 \mathrm{mM}$ EDTA. Protein concentrations were determined using the bicinchoninic acid protein assay and samples were diluted to a concentration of $2 \mu \mathrm{g} / \mu \mathrm{l}$. Protein samples $(40 \mu \mathrm{g})$ were then separated by $12 \%$ SDS-PAGE. The separated proteins were transferred onto polyvinylidene difluoride membranes and then blocked with confining liquid (5\% skimmed milk powder and $0.1 \%$ Tween-20 in PBS) at room temperature for $2 \mathrm{~h}$, and incubated overnight at $4^{\circ} \mathrm{C}$ with primary antibody: Rabbit anti-caspase- 3 and $\beta$-actin antibodies (004F and ab8226, 1:1,000 dilution; Abcam) in hybridization solution. The following day, the membranes were incubated with secondary antibody (goat-anti-rabbit immunoglobulin G, S001F, 1:2,000 dilution; Abcam) in hybridization solution at room temperature for $2 \mathrm{~h}$. Blots were visualized by enhanced chemoluminescence (EMD Millipore, Billerica, MA, USA) was used for signal detection. Images were quantified using the Quantity One software (v4.62; Bio-Rad Laboratories, Inc., Hercules, CA, USA). Values are expressed as the optical density ratio of the target protein to $\beta$-actin.

Statistical analysis. Values are expressed as the mean \pm standard deviation and were analyzed using SPSS 19.0 statistical software (IBM Corp., Armonk, NY, USA). The data were analyzed by one-way ANOVA and further analyzed by Dunnett's T3 test for multiple comparisons. A two-tailed $\mathrm{P}<0.05$ was considered to indicate a statistically significant difference.

\section{Results}

CGRP affects EEG in rats after heat stroke. Compared with the control group, a rapid suppression of the rat EEG was observed in the HS group. A representative slow wave pattern appeared. The amplitude of the $\theta$-wave increased significantly $(\mathrm{P}<0.05)$ and the amplitude of the $\alpha$-wave decreased significantly $(\mathrm{P}<0.05)$. Administration of CGRP produced a rapid 
Table I. Comparison of electroencephalogram parameters $(\mu \mathrm{V})$ for rats in different group.

\begin{tabular}{|c|c|c|c|c|}
\hline Group & $\delta$-wave & $\theta$-wave & $\alpha$-wave & $\beta$-wave \\
\hline Control & $44.40 \pm 12.50$ & $30.20 \pm 3.49$ & $35.60 \pm 6.84$ & $8.80 \pm 3.56$ \\
\hline HS & $60.80 \pm 6.90$ & $54.00 \pm 10.86^{\mathrm{a}}$ & $17.40 \pm 4.45^{\mathrm{a}}$ & $10.80 \pm 4.15$ \\
\hline HS+CGRP & $52.00 \pm 8.37$ & $35.50 \pm 5.70^{\mathrm{b}}$ & $28.80 \pm 4.82^{\mathrm{b}}$ & $9.00 \pm 2.24$ \\
\hline HS+CGRP8-37 & $65.00 \pm 7.91^{\mathrm{a}}$ & $70.00 \pm 11.73^{\mathrm{a}}$ & $20.40 \pm 2.61^{\mathrm{a}}$ & $10.00 \pm 2.45$ \\
\hline
\end{tabular}

${ }^{\mathrm{a}} \mathrm{P}<0.05$ vs. control group; ${ }^{\mathrm{b}} \mathrm{P}<0.05$ vs. HS group. Values are expressed as the mean \pm standard deviation ( $=5 /$ group). CGRP, calcitonin gene-related peptide; HS, heat stroke.
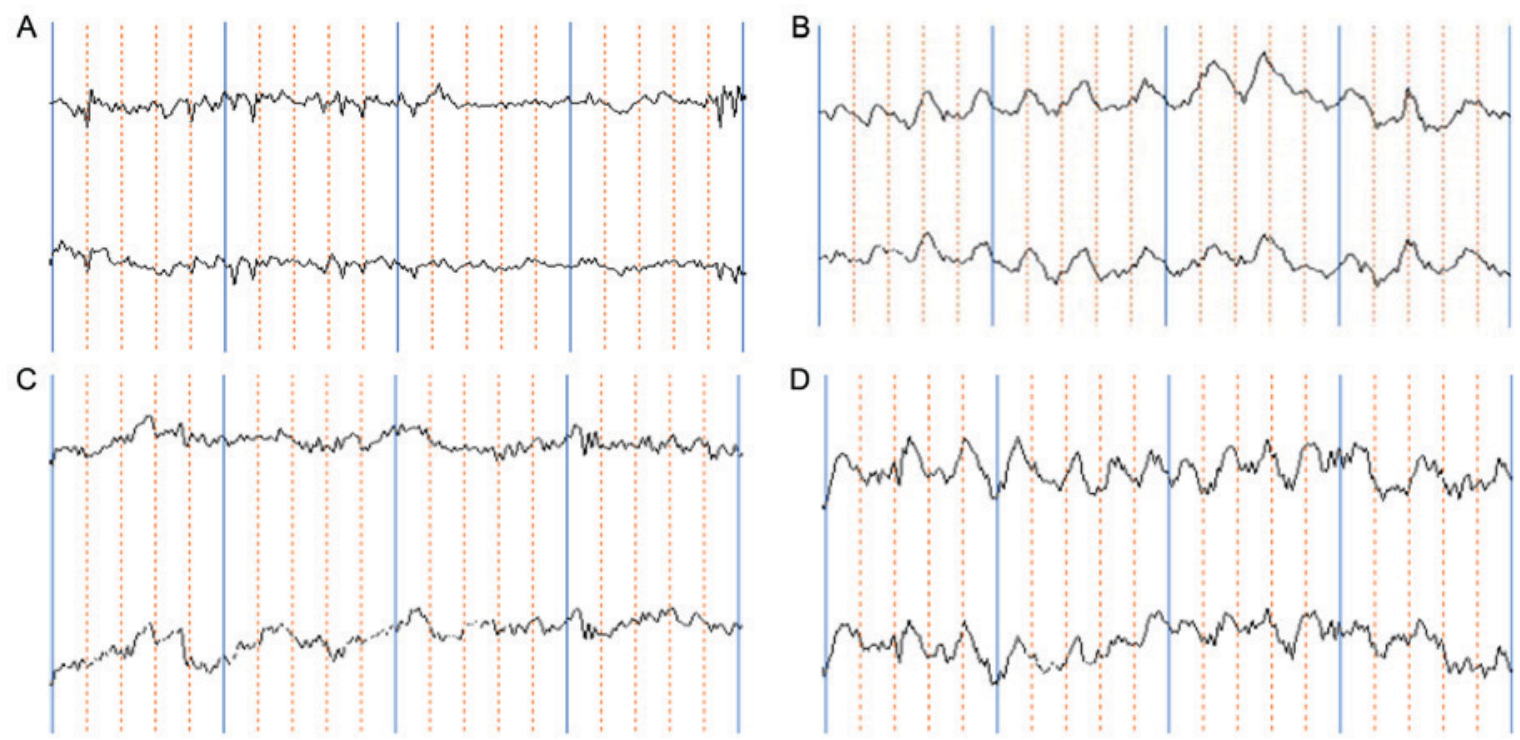

Figure 1. EEG waves of rats in each group. (A) Control group, normal number of short-term fast waves. (B) HS group, large number of long-term slow waves. (C) HS+CGRP group, rapid recovery of EEG. (D) HS+CGRP8-37 group, great suppression in the duration of EEG. EEG, electroencephalogram; HS, heat stroke; CGRP, calcitonin gene-related peptide.

recovery of the EEG. The EEG was characterized by short- to long-term $\alpha$-waves and low-voltage $\beta$-waves as well as a large amount of intermittent $\delta$ - and $\theta$-waves. Compared with that in the HS group, the $\theta$-wave decreased significantly $(\mathrm{P}<0.05)$ while the $\alpha$-wave increased significantly in the CGRP group $(\mathrm{P}<0.05)$. CGRP8-38 produced a great suppression in the duration of the EEG. The characteristics of the EEG were a long-term frequency $\theta$-wave, inclusion of a partial $\delta$-wave and a significant reduction in the $\alpha$-wave. Compared with those in the control group, the $\theta$ - and $\delta$-wave increased significantly $(\mathrm{P}<0.05)$, while the $\alpha$-wave decreased significantly $(\mathrm{P}<0.05)$. However, these differences did not achieve any statistical significance when compared with the HS group (Table I; Fig. 1).

CGRP alleviates brain tissue damage after heat stroke. Histopathological investigation revealed that no significant abnormalities were present in the brains of control rats. By contrast, the brain exhibited moderate edema, characterized by vacant spaces surrounding the neurons and capillaries in HS rats. Pathological aberrations in the brains of rats in the HS+CGRP group were significantly alleviated. Nerve cells only exhibited slight swelling and the gaps between the nerves and blood vessels were displayed. Severe damage was observed in the HS+CGRP8-37 group. Neural cell shrinkage, volume reduction, hyperchromatic nuclei, nuclear pyknosis, disappearance of part of the nuclear membrane and cell necrosis were observed in the HS+CGRP8-37 group (Fig. 2).

CGRP reduces heat stroke-induced neuronal cell apoptosis. As presented in Figs. 3 and 4, heat stroke significantly enhanced neuronal cell apoptosis as compared with that in the control group $(\mathrm{P}<0.05)$. The heatstroke-induced upregulation of cell apoptosis was significantly weakened after administration of CGRP. The number of TUNEL-positive cells in the HS+CGRP group was significantly lower than that in the HS group $(\mathrm{P}<0.05)$. By contrast, CGRP8-37 enhanced neuronal cell apoptosis. The results indicated a significantly enhanced number of TUNEL-positive cells in the CGRP8-37 group compared with that in the HS group $(\mathrm{P}<0.05)$. As indicated by these data, GRP and CGRP8-37 affected neuronal cell apoptosis. It may be deduced that CGRP contributed to the inhibitory effects of neuronal cell apoptosis induced by heat stroke.

CGRP inhibits heat stroke-induced expression of caspase-3 protein in the brains of rats. Western blot 
A

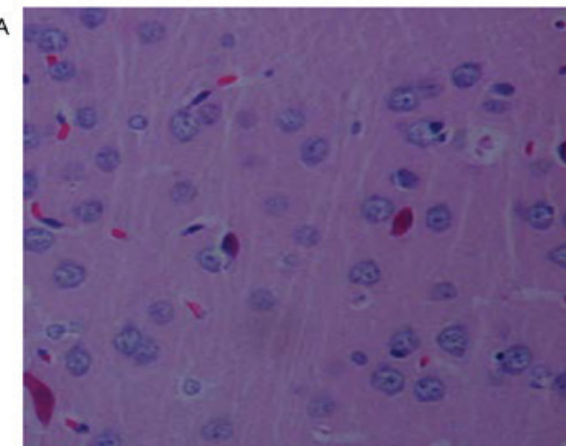

C

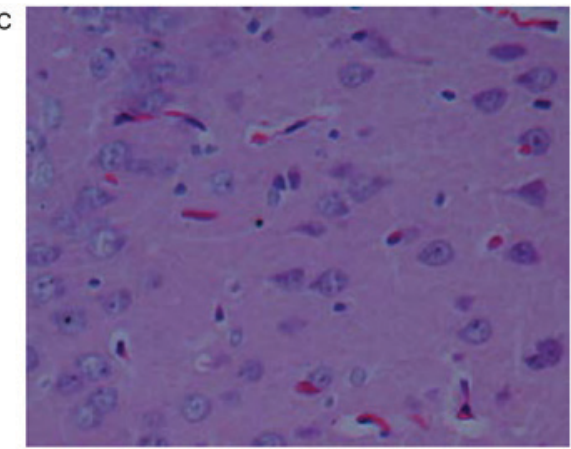

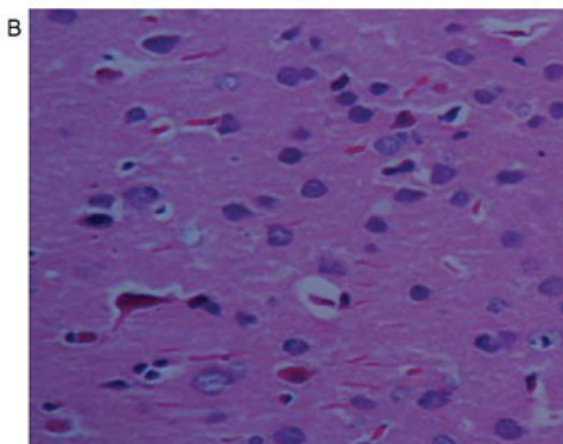

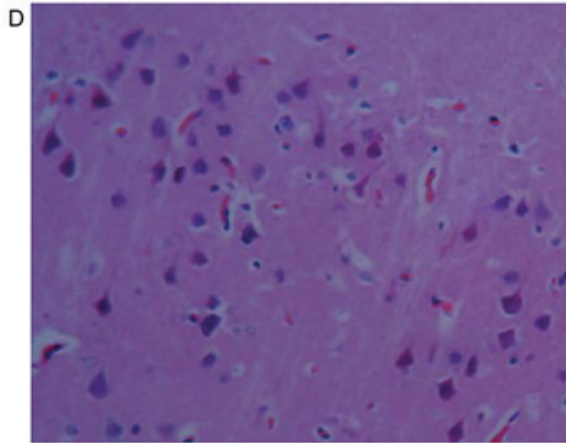

Figure 2. Hematoxylin and eosin staining of rat brain tissue in each group (magnification, x400). (A) Control group, normal morphology of neurons. (B) HS group, moderate neuronal swelling and widening of peri-cellular spaces. (C) HS+CGRP group, slight neuron swelling and vacant spaces surrounding the neurons and capillaries. (D) HS+CGRP8-37 group, obvious neuron shrinkage. HS, heat stroke; CGRP, calcitonin gene-related peptide.
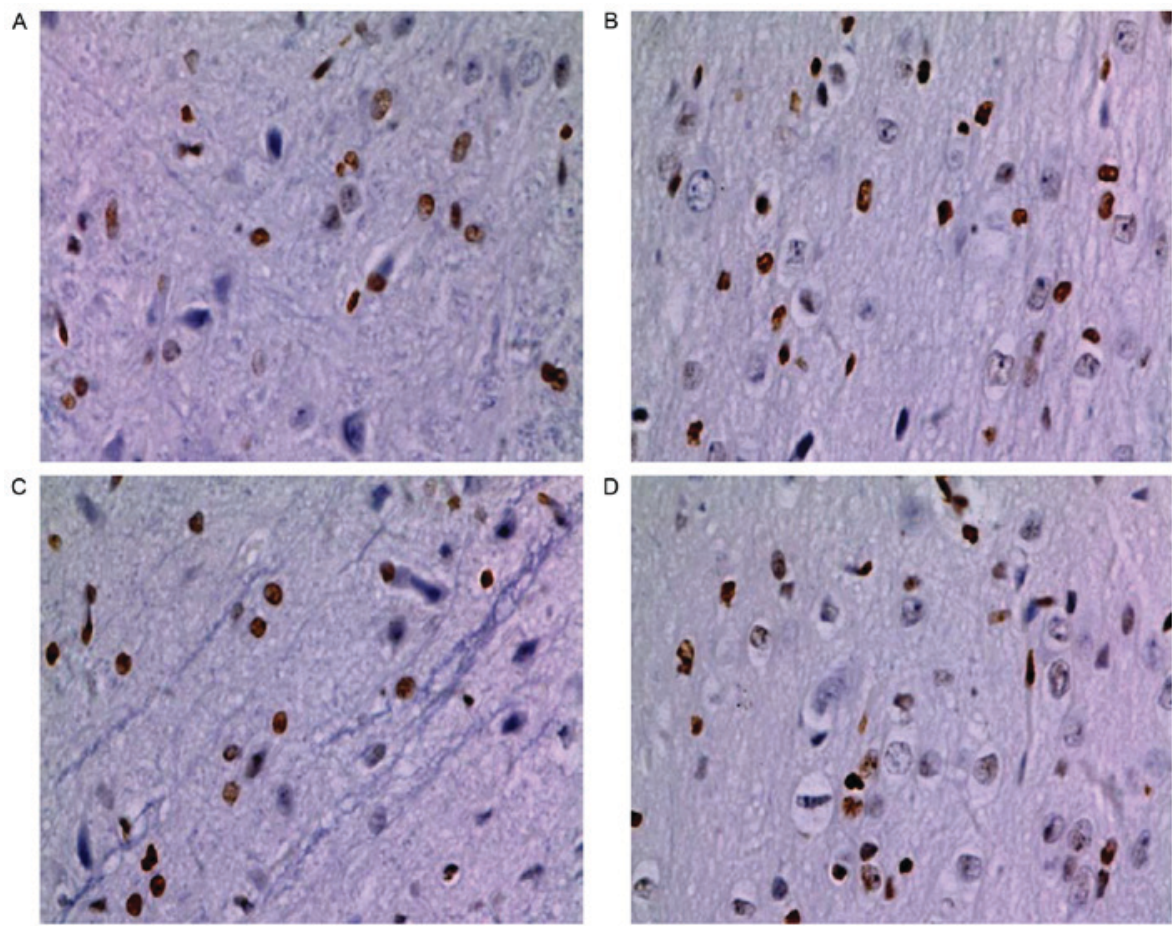

Figure 3. Apoptosis of rat brain tissue in each group measured by TUNEL (magnification, x200). (A) Control group, small amount of TUNEL-positive cells. (B) HS group, significant amount of TUNEL-positive cells. (C) HS+CGRP group, TUNEL-positive cells significantly decreased compared with those in B. (D) HS+CGRP8-37 group, significant amount of TUNEL-positive cells. TUNEL, terminal deoxynucleotidyltransferase-mediated deoxyuridine triphosphate nick end labelling; HS, heat stroke; CGRP, calcitonin gene-related peptide.

analysis demonstrated that all rats subjected to heat stroke demonstrated a significant increase in the protein levels of caspase- 3 when compared with those in the control group $(\mathrm{P}<0.05)$. Administration of CGRP significantly prevented the increase of caspase- 3 protein expression. The protein levels of caspase-3 in the HS+CGRP group significantly decreased compared with those in the HS group $(\mathrm{P}<0.05)$. By contrast, there was an apparent increase in the expression 


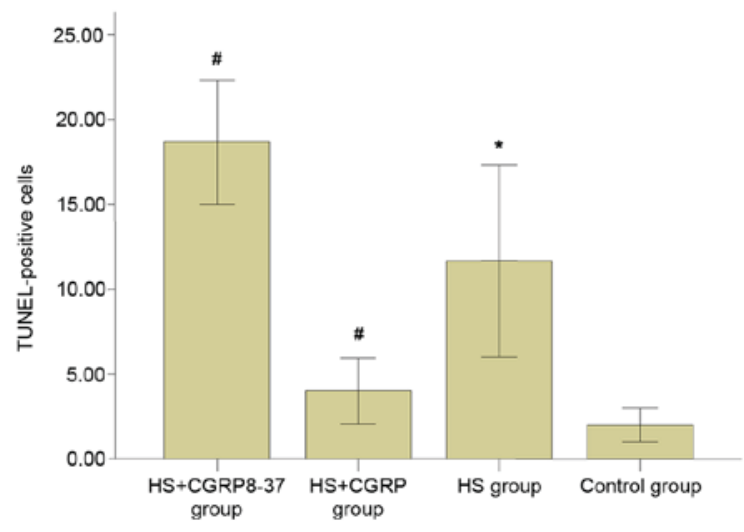

Figure 4. Comparison of apoptosis in rat brain tissue in each group. TUNEL-positive cells were decreased in CGRP group. The apoptosis was highest in HS+CGRP8-37 group. ${ }^{~} \mathrm{P}<0.05$ vs. control group; ${ }^{\text {" }} \mathrm{P}<0.05$ vs. HS group. HS, heat stroke; TUNEL, terminal deoxynucleotidyltransferasemediated deoxyuridine triphosphate nick end labelling; CGRP, calcitonin gene-related peptide.

A

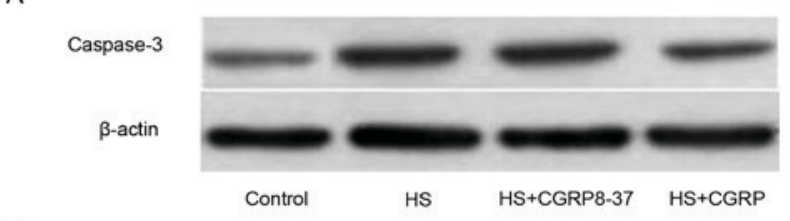

B

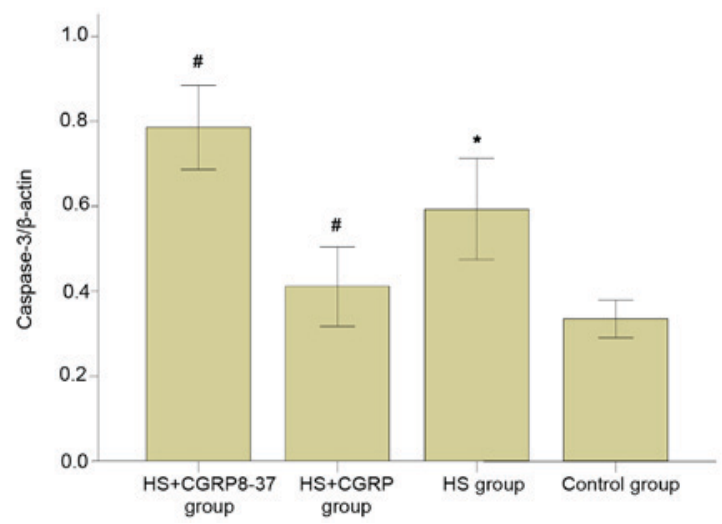

Figure 5. Expression of caspase-3 in brain tissue of rats from each group measured by western blot analysis. (A) Representative western blot analysis (B) Quantified expression of caspase-3 relative to $\beta$-actin in each group Expression of caspase-3 protein was inhibited by CGRP, while elevated by CGRP8-37 as compared with HS group. ${ }^{*} \mathrm{P}<0.05$ vs. control group; ${ }^{\#} \mathrm{P}<0.05$ vs. HS group. CGRP, calcitonin gene-related peptide; HS, heat stroke.

of caspase-3 protein in the brains of rats in the CGRP8-37 group compared with those in the HS group ( $\mathrm{P}<0.05$; Fig. 5). It was revealed that CGRP8-37 significantly enhanced the protein levels of caspase- 3 in brain tissues. These results indicated that the expression of caspase- 3 protein induced by heat stroke was considerably downregulated by CGRP protein in the brains of rats.

\section{Discussion}

According to its pathophysiological responses, heat stroke may be defined as a form of hyperthermia, associated with a systemic inflammatory response, leading to a syndrome of multi-organ dysfunction in which central nervous system injury dominates $(10,11)$. Heat-associated illnesses develop when the pathological effects of heat load are not prevented. Syndromes vary from less severe, such as heat syncope, to severe forms, such as lethal heat stroke. Knowledge of the pathological changes of the central nervous system is essential for understanding the mechanisms of heat stroke. However, at present, little information regarding the occurrence of brain lesions is available. Bouchama et al (12) demonstrated that tissue damage of varying degree was present in the brain after heat stroke. In a rat model of heat stroke, injury to the brain appeared even at $39^{\circ} \mathrm{C}$, which was further aggravated with increases in temperature, regardless of cooling treatment (13). However, the mechanism of brain injury in heat stroke still remain elusive, and effective methods to prevent the progression of brain injury in the clinic are currently lacking. CGRP, a newly identified endogenous neural active peptide, is widely distributed in the nervous system and has been considered to have a positive role in central nervous system injury in this decade (14-16). Increasing evidence has indicated that CGRP has a protective effect on non-septic brain injury, such as cerebral infarction, subarachnoid hemorrhage and traumatic brain injury. In a rat model, CGRP reduced the degree of cerebral ischemia/reperfusion injury and improved the tolerance of nerve cells to hypoxia (6). Animal and clinical experiments have indicated that CGRP also relieves cerebral vasospasm after subarachnoid hemorrhage, improves the blood supply of the brain and regulates the circulation of the brain (17). Previous studies have indicated a sharp release of CGRP from the brain tissue into the serum after traumatic brain injury (5). Growing evidence has also suggested that CGRP is an important mediator in septic and sepsis-like brain injury. Studies have demonstrated that the expression of central CGRP was significantly increased in a sepsis model and a scald model $(18,7)$. Considering that the mechanism of brain injury after heat stroke is similar to that of sepsis, the present study hypothesized that CGRP is also a potential protective factor in brain injury after heat stroke. To the best of our knowledge, no studies are available that explored the protective effects of CGRP on brain injury induced by heat stroke.

To observe the potential protective effect of CGRP against brain injury in heat stroke, CGRP and CGRP8-37 were respectively injected into the rats after heat stroke through the carotid artery trocar. The results indicated that brain injury was present at an early stage after heat stroke and CGRP exerted a protective effect on brain injury at the same time-point, as directly evidenced by techniques such as EEG and histopathology. EEG examination has the characteristics of high sensitivity and fastness. In the present study, changes in the EEG were examined in an attempt to observe the effect of CGRP on brain injury after heat stroke. An extensive slow wave is the typical change in the EEG after diffuse brain injury. The degree of the slow wave is linked to the severity of brain damage (19). In the present study, heat stroke evidently induced an abnormal EEG, which manifested as a large quantity of representative slow waves, increased amplitude of $\theta$-wave and decreased amplitude of $\alpha$-wave. Administration of CGRP produced a rapid recovery of the electroencephalogram. The appearance of a reverse tendency after bolus 
injection of CGRP8-37 further confirmed the brain-protective effect of CGRP. H\&E staining results further evidenced the protective effects of CGRP on brain injury. Compared with those in the HS group, pathological brain damage was significantly alleviated in the HS+CGRP group and enhanced in the HS+CGRP8-37 group, which indicated that CGRP may have a marked protective effect in heat stroke. In fact, CGRP is the most potent microvascular neuropeptide vasodilator known to date $(20,21)$. Previous studies have demonstrated that exogenous CGRP significantly increased the cerebral blood flow, prevented blood-brain barrier injury and protected ischemic neurons in cerebrovascular disease $(22,6)$. The results of the present study were consistent with those of studies on ischemic brain injury, which suggested the presence of common mechanisms. Associated studies have reported that heat stress exerts cardioprotection, which is due to the synthesis and release of CGRP via activation of capsaicin receptor (vanilloid receptor subtype 1) on capsaicin-sensitive sensory neurons. The endothelial cell-derived CGRP is considered as one of the key factors exerting protective effects (23). It remains elusive whether a similar mechanism may explain the protective effect of CGRP on the central nervous system in sepsis-like heat stroke.

Clinical and laboratory studies have confirmed that neuronal apoptosis is one of the important causes of brain damage in various types of brain injury. Apoptosis has a dual effect: While it may clear aging cells and exert a protective effect on tissues and organisms, excessive apoptosis may lead to massive neuronal loss and then damage the nervous function, causing secondary damage to the brain. With the continuous deepening of the understanding of the damage heat stroke causes to the body, an increasing number of studies have indicated that heat stroke also induces apoptosis of brain neurons $(13,24)$. Whether the effect of CGRP in brain damage induced by severe heat stroke is associated with its regulation of the apoptosis of cells of the central nervous system had remained to be determined. For this reason, the present study assessed neuronal cell apoptosis in rats. The results revealed that the heat stroke-induced upregulation of cell apoptosis was significantly weakened after administration of CGRP. Conversely, treatment with CGRP8-37 enhanced neuronal cell apoptosis. From these results, it may be deduced that CGRP exerted an inhibitory effect on neuronal cell apoptosis induced by heat stroke. CGRP is known to activate signal transduction pathways through the $G$ protein-coupled receptor. Subsequently, the accumulation of intracellular cyclic adenosine monophosphate leads to changes of cell function, including apoptosis mediated through complex signaling pathways. Caspase- 3 protein has a key role in the process of apoptosis and it is the most common downstream effector of apoptotic pathways $(25,26)$. Associated studies have indicated that the cytoprotective role of CGRP (i.e., its anti-apoptotic effect) after ischemia/reperfusion injury of the heart, brain and gastrointestinal system is exerted via downregulation of caspase- 3 by modulating nitric oxide production $(27,28)$. The present study explored the possible molecular mechanisms of the effect of CGRP. It was examined whether CGRP regulated caspase- 3 protein levels in rats after heat stroke. The results indicated that the level of caspase- 3 in rats with heat stroke was significantly decreased following a bolus injection of
CGRP, while it was apparently increased after administration of CGRP8-37. It was therefore suggested that CGRP exerted its inhibitory effects on apoptosis in rat brains interfering with caspase-3 signaling. The present results are comparable with those of previous studies. However, it remains elusive which upstream signaling proteins are directly affected to promote this downregulation of caspase- 3 and additional research is required to clarify the exact mechanism.

Overall, the results of the present study demonstrated that CGRP has a protective effect on early-stage brain injury induced by heat stroke in rats. Furthermore, it was demonstrated that the underlying mechanism of the protective effect of CGRP includes inhibition of neuronal cell apoptosis via downregulation of caspase-3. The present study provided novel insight into the role of CGRP in heat stroke, but had certain limitations. The damage in the brain was detected at a single time-point after heat stroke, and no longer-term observation was performed. Due to unpredictability, administration of CGRP is less practical in the clinic; therefore, further study is required to explore safer methods. In brief, the exact mechanism via which CGRP reduces central nervous system injury after heat stroke remains to be assessed to provide a theoretical basis for the treatment of heat stroke by CGRP.

\section{Acknowledgements}

This study was supported in part by grants from the National Natural Science Foundation of China (grant nos. 81071529 and 81272105).

\section{References}

1. Leon LR: Hypothermia in systemic inflammation: Role of cytokines. Front Biosci 9: 1877-1888, 2004.

2. Brownlow MA, Dart AJ and Jeffcott LB: Exertional heat illness: A review of the syndrome affecting racing Thoroughbreds in hot and humid climates. Aust Vet J 94: 240-247, 2016.

3. Székely M, Carletto L and Garami A: The pathophysiology of heat exposure. Temperature (Austin) 2: 452, 2015.

4. Zhang D, Zhang P, Wang Y, Han N, Tang C and Jiang B: The influence of brain injury or peripheral nerve injury on calcitonin gene-related peptide concentration variation and fractures healing process. Artif Cells Blood Substit Immobil Biotechnol 37: 85-91, 2009.

5. Song Y, Bi L, Zhang Z, Huang Z, Hou W, Lu X, Sun P and Han Y: Increased levels of calcitonin gene-related peptide in serum accelerate fracture healing following traumatic brain injury. Mol Med Rep 5: 432-438, 2012.

6. Liu Z, Liu Q, Cai H, Xu C, Liu G and Li Z: Calcitonin gene-related peptide prevents blood-brain barrier injury and brain edema induced by focal cerebral ischemia reperfusion. Regul Pept 171: 19-25, 2011

7. Hu D, Chen B, Wang B and Ma D: The changes in the morphology and distribution of substance $\mathrm{P}$ and calcitonin-gene related peptide nerves in the anterior pituitary of scalded rats. Zhonghua Shao Shang Za Zhi 18: 176-179, 2002 (In Chinese).

8. Satoh M, Kuraishi Y and Kawamura M: Effects of intrathecal antibodies to substance $\mathrm{P}$, calcitonin gene-related peptide and galanin on repeated cold stress-induced hyperalgesia: Comparison with carrageenan-induced hyperalgesia. Pain 49: 273-278, 1992.

9. Legat FJ, Jaiani LT, Wolf P, Wang M, Lang R, Abraham T, Solomon AR, Armstrong CA, Glass JD and Ansel JC: The role of calcitonin gene-related peptide in cutaneous immunosuppression induced by repeated subinflammatory ultraviolet irradiation exposure. Exp Dermatol 13: 242-250, 2004.

10. Bouchama A: Heatstroke: Facing the threat. Crit Care Med 34: 1272-1273, 2006 .

11. Leon LR and Helwig BG: Heat stroke: Role of the systemic inflammatory response. J Appl Physiol (1985) 109: 1980-1988, 2010. 
12. Bouchama A, Roberts G, Al Mohanna F, El-Sayed R, Lach B, Chollet-Martin S, Ollivier V, Al Baradei R, Loualich A, Nakeeb S, et al: Inflammatory, hemostatic, and clinical changes in a baboon experimental model for heatstroke. J Appl Physiol (1985) 98: 697-705, 2005

13. Liu ZF, Li BL, Tong HS, Tang YQ, Xu QL, Guo JQ and Su L: Pathological changes in the lung and brain of mice during heat stress and cooling treatment. World J Emerg Med 2: 50-53, 2011.

14. Edvinsson L: The Journey to establish CGRP as a migraine target: A retrospective view. Headache 55: 1249-1255, 2015.

15. Schebesch KM, Herbst A, Bele S, Schödel P, Brawanski A Stoerr EM, Lohmeier A, Kagerbauer SM, Martin J and Proescholdt M: Calcitonin-gene related peptide and cerebral vasospasm. J Clin Neurosci 20: 584-586, 2013.

16. Kageneck C, Nixdorf-Bergweiler BE, Messlinger $\mathrm{K}$ and Fischer MJ: Release of CGRP from mouse brainstem slices indicates central inhibitory effect of triptans and kynurenate. J Headache Pain 15: 7, 2014

17. Tian XH, Wang ZG, Meng H, Wang YH, Feng W, Wei F, Huang ZC, Lin XN and Ren L: Tat peptide-decorated gelatin-siloxane nanoparticles for delivery of CGRP transgene in treatment of cerebral vasospasm. Int J Nanomedicine 8: 865-876, 2013.

18. De winter BY, Bredenoord AJ, Van Nassauw L, De Man JG, De Schepper HU, Timmermans JP and Pelckmans PA: Involvement of afferent neurons in the pathogenesis of endotoxin-induced ileus in mice: Role of CGRP and TRPV1 receptors. Eur J Pharmacol 615: 177-184, 2009.

19. Sinha RK: Electro-encephalogram disturbances in different sleep-wake states following exposure to high environmental heat. Med Biol Eng Comput 42: 282-287, 2004.

20. Smillie SJ and Brain SD: Calcitonin gene-related peptide (CGRP) and its role in hypertension. Neuropeptides 45: 93-104, 2011.

21. Mai TH, Wu J, Diedrich A, Garland EM and Robertson D Calcitonin gene-related peptide (CGRP) in autonomic cardiovascular regulation and vascular structure. J Am Soc Hypertens 8: 286-296, 2014.
22. Zhang ZH, Fang XB, Xi GM, Li WC, Ling HY and Qu P: Calcitonin gene-related peptide enhances CREB phosphorylation and attenuates tau protein phosphorylation in rat brain during focal cerebral ischemia/reperfusion. Biomed Pharmacother 64: 430-436, 2010.

23. Ye F, Deng PY, Li D, Luo D, Li NS, Deng S, Deng HW and $\mathrm{Li}$ YJ: Involvement of endothelial cell-derived CGRP in heat stress-induced protection of endothelial function. Vascul Pharmacol 46: 238-246, 2007.

24. Kibayashi K, Nakao K and Shojo H: Hyperthermia combined with ethanol administration induces $\mathrm{c}$-fos expression in the central amygdaloid nucleus of the mouse brain. A possible mechanism of heatstroke under the influence of ethanol intake. Int J Legal Med 123: 371-379, 2009.

25. Ji J, Kline AE, Amoscato A, Samhan-Arias AK, Sparvero LJ, Tyurin VA, Tyurina YY, Fink B, Manole MD, Puccio AM, et al: Lipidomics identifies cardiolipin oxidation as a mitochondrial target for redox therapy of brain injury. Nat Neurosci 15: 1407-1413, 2012.

26. Rao RV, Hermel E, Castro-Obregon S, del Rio G, Ellerby LM, Ellerby HM and Bredesen DE: Coupling endoplasmic reticulum stress to the cell death program. Mechanism of caspase activation. J Biol Chem 276: 33869-33874, 2001.

27. Duan L, Lei H, Zhang Y, Wan B, Chang J, Feng Q and Huang W: Calcitonin gene-related peptide improves hypoxia-induced inflammation and apoptosis via nitric oxide in $\mathrm{H} 9 \mathrm{c} 2$ cardiomyoblast cells. Cardiology 133: 44-53, 2016.

28. Luo CC, Huang CS, Ming YC, Chu SM and Chao HC: Calcitonin gene-related peptide downregulates expression of inducible nitride oxide synthase and caspase-3 after intestinal ischemia-reperfusion injury in rats. Pediatr Neonatol 57: 474-479, 2016. 